\title{
Focus on small lesion of alpha-fetoprotein producing gastric cancer
}

\author{
Zhe Shen $\cdot$ Chaohui Yu $\cdot$ Youming Li
}

Accepted: 4 October 2010 /Published online: 21 October 2010

(C) Springer-Verlag 2010

\section{Dear Editor:}

Alpha-fetoprotein (AFP) remains a clinically meaningful instrument for diagnosis of hepatocellular carcinoma (HCC). We meet a female patient with AFP-producing gastric cancer (AFP-GC) accompanied with gallbladder adenomyosis; she was misdiagnosed with gallbladder cancer in the beginning.

A 50-year-old woman complained of abdominal distension and fatigue for 3 months. She underwent an ultrasonographic examination and was diagnosed with adenomyomatosis of the gallbladder. Also, she underwent a gastroscopy with biopsies and was diagnosed with ulcer of gastric cardia $(0.7 \times 0.5 \mathrm{~cm})$ accompanied with Helicobacter pylori infection; so she accepted quadruple therapy. Her serum AFP level $(43.8 \mathrm{ng} / \mathrm{ml})$ was slightly higher than the reference value. As the AFP was positive, associated with adenomyomatosis of gallbladder, she came to our hospital for further diagnosis. Abdominal computerized tomography showed adenomyomatosis of the gallbladder. However, AFP level $(74.3 \mathrm{ng} / \mathrm{ml})$ was higher than the previous level. Based on the above results, she decided to accept laparoscopic cholecystectomy, and pathology confirmed adenomyomatosis of gallbladder. Unfortunately, after quadruple therapy and cholecystectomy, she still felt abdominal distension and fatigue. Gastroscopy was performed and showed that gastric angle ulcer did not heal, and pathology confirmed carcinoma of gastric cardia. Simultaneously, AFP level $(96.4 \mathrm{ng} / \mathrm{ml})$ was obviously higher than the previous two tests. She accepted gastrectomy surgery, and fortunately, there was no peripheral lymphnode malignant metastasis. After six courses of chemotherapy, AFP level $(4.5 \mathrm{ng} / \mathrm{ml})$ returned to normal.

Alpha-fetoprotein (AFP) is a serum protein, which is synthesized by fetal liver, yolk sac cells, and fetal gastrointestinal cells. Actually, AFP is evaluated as it is one of most important serum marker for early stage HCC, clinically. AFP-GC has an aggressive behavior with the lymphatic system, is hematogenous, and metastasizes into the liver at an early stage. After cholecystectomy, if we did not follow up the AFP levels of the patient, she could be misdiagnosed or liver metastasis could have occurred when she came to hospital again. Due to poor prognosis of AFP-GC, clinicians need to pay more attention to the diagnosis. When we encountered patients with elevated AFP, we need to focus on AFP-GC except HCC.

Conflict of interest The authors declare that they have no conflict of interest.

\footnotetext{
Z. Shen $\cdot$ C. Yu $\cdot$ Y. Li $(\bowtie)$

Department of Gastroenterology, The First Affiliated Hospital,

College of Medicine, Zhejiang University,

Qingchun Road 79\#,

Hangzhou 310003, China

e-mail: shenzhe39@126.com
} 